\title{
Dexmedetomidine Alleviates Hyperalgesia in Arthritis Rats Through Inhibition of the p38MAPK Signaling Pathway
}

\section{Bin Nie}

Tongji Hospital in Wuhan, Tongji Medical College, Huazhong University of Science and Technology Hui Jiang

Tongji Hospital in Wuhan, Tongji Medical College, Huazhong University of Science and Technology Hong Chen

Tongji Hospital in Wuhan, Tongji Medical College, Huazhong University of Science and Technology Qiong Liu ( $\nabla$ qiongl0514@163.com )

Tongji Hospital in Wuhan, Tongji Medical College, Huazhong University of Science and Technology https://orcid.org/0000-0003-1292-6903

\section{Research Article}

Keywords: Arthritis, Dexmedetomidine, Hyperalgesia, p38MAPK signaling pathway, Inflammation

Posted Date: November 9th, 2021

DOI: https://doi.org/10.21203/rs.3.rs-1049464/v1

License: (9) This work is licensed under a Creative Commons Attribution 4.0 International License. Read Full License

Version of Record: A version of this preprint was published at Immunopharmacology and Immunotoxicology on April 21st, 2022. See the published version at https://doi.org/10.1080/08923973.2022.2069578. 


\section{Abstract}

Background: Dexmedetomidine (DEX) has showed significant analgesic effects in neuropathic pain, but the underlying mechanism has remained elusive. Our present study aimed to explore the effect of DEX on hyperalgesia with the involvement of p38MAPK signaling pathway a rat model of monoarthritis (MA).

Methods: MA rat model was induced by injection of Complete Freund's Adjuvant (CFA). Pathological changes of ma rats were observed by HE staining and Safranin-O/Fast Green staining. Ankle circumference, paw withdrawal latency (PWL) and paw withdrawal threshold (PWT) was measured to judge the degree of hyperalgesia in MA rats. Immunohistochemistry and ELISA were applied to observe the degree of inflammation in rats. Western blot analysis was conducted to detect expression of p38MAPK signaling pathway-related factors. The mechanism of p38MAPK signaling pathway in MA rats was observed via treatment of Anisomycin or SB203580 combined with DEX.

Results: After $8 \mathrm{~h}$ of CFA induction, joint swelling and hyperalgesia occurred in rats. There were obvious pathological changes in the joint cavity, the joint cavity space became narrow and synovial bursa became rough. A large number of inflammatory cell infiltration was observed under microscope. After injection of DEX and SB203580, PWT and PWL was prolonged, the expression of serum inflammatory factors was decreased, and the expression of p38MAPK signaling pathway-related factors was decreased; while all the detected indexes were recovered in MA rats after treated with DEX and Anisomycin.

Conclusions: Our study provided evidence that DEX could alleviate hyperalgesia in arthritis rats through inhibition of the p38MAPK signaling pathway.

\section{Introduction}

Arthritis is a process of joint dysfunction, with acute or long-term inflammation that affects one or more joints of the patient, and the most common types of arthritis are osteoarthritis (OA) and rheumatoid arthritis (RA) [1]. The well-known symptoms of arthritis include pain, swelling, joint stiffness and eroded cartilage [2]. To be specific, arthritis may further lead to a pain syndrome characterized by spontaneous pain, allodynia and hyperalgesia [3]. Multiple kinds of factors were reported to be correlated with an increased risk of arthritis, including aging, obesity, joint injury and genetic factors as well as abnormal reactions of the immune system [4]. Dexmedetomidine (DEX), a selective a2-adrenoceptor (a2-AR) agonist, has been found to exert significant analgesic effects on acute inflammatory pain and postoperative pain $[5,6]$. a2-AR is preponderantly expressed in vasculature and central nervous system, and moreover, the presence of a2-AR in cells with sympathetic reactive responses including adipocytes, pancreatic $\beta$-cells, and cancer epithelial cells can be observed [7]. Activation of a2-AR inhibits the transmission of pain input to the higher axis, thus reducing the hyperactivity of spinal dorsal horn neurons in neuropathic pain [8]. Previous evidence demonstrated that DEX administration created a dosedependent antihyperalgesic effect in complete Freund's adjuvant (CFA)-induced monoarthritis (MA) rats, suggesting that a2AR also played a crucial role in regulating pain produced by inflammation [9]. A 
randomized clinical trial has demonstrated that intra-articular injection of DEX can provide rapid and long-term relief of knee pain and swelling in RA patients [10].

p38 mitogen-activated protein kinases (p38MAPK) is a well-known signaling pathway in the cell, and is involved in various kinds of biological processes, including cell apoptosis and cell movement as well as plays a key role in mediating inflammation and cytokine generation [11]. Evidence showed that the timely inactivation of the $\mathrm{p} 38 \mathrm{MAPK}$ signaling pathway was necessary to inhibit excessive inflammatory responses [12]. The CFA-induced MA rat model, which shows many of the pathological features of RA, has been widely deemed as a laboratory model in the study of arthritic pain [9]. Besides, the p38MAPK signaling pathway has been closely related to the pathology of RA, specifically, it has been thought to be a promising target for finding new anti-inflammatory drugs to treat RA and other chronic immunemediated inflammatory diseases [13]. However, the molecular mechanism underlying the anti-nociceptive effects of DEX on arthritis with the involvement of the P38MAPK signaling pathway has not been fully uncovered. The present study aimed to investigate whether injection with DEX has anti-nociceptive effects through inhibition of the p38MAPK signaling pathway in rats with CFA-induced MA.

\section{Materials And Methods}

\section{Experimental animals}

A total of 50 healthy adult male Wistar rats $(150 \mathrm{~g}-200 \mathrm{~g})$ were selected and fed under 12-h dark and 12-h light conditions with free access to food and water. This study was approved and supervised by the animal ethics committee of TongJi Hospital in Wuhan, Tongji Medical College, Huazhong University of Science and Technology. Significant efforts were made in order to minimize both the number of animals and their suffering. All procedures were strictly conducted in accordance with the codes of International Association for the Study of Pain.

\section{Model establishment and grouping}

After the rats were anesthetized by intraperitoneal injection of $10 \%$ urethane $(1 \mathrm{~g} / \mathrm{kg})$, a volume of $50 \mu \mathrm{L}$ complete Freund's adjuvant (CFA) (Sigma, St Louis, MO, USA) was injected into ankle articular cavity. After 4-8 $\mathrm{h}$, the local inflammatory response and the pain-induced sensitization of the injection joint were observed. Rats injected with sterile normal saline (NS) were regarded as the sham group. The 85 rats were grouped as follows: sham (10 rats), MA (10 rats), MA + NS (10 rats), MA + DEX (40 rats), MA + SB203580 (5 rats), MA + Anisomycin (5 rats), and MA + Anisomycin/DEX groups (5 rats). According to the DEX concentration, the MA + DEX group was further divided into 4 groups $(2.5,5,10,20 \mu \mathrm{g} / \mathrm{kg})$ and normal saline (NS) group, with 10 rats in each group. DEX was injected intraperitoneally on the third day after MA modeling. The changes of pain sensitivity in rats were detected 15,30,45, 60,90, 120, and 150 min after administration. After induction of CFA, SB203580 (Sigma) and Anisomycin (Sigma) $(5 \mathrm{mg} / \mathrm{kg}$, dissolved in $5 \mathrm{mg} / \mathrm{mL}$ DMSO) were intraperitoneally injected for three days, and the control rats were injected with the same dose of DMSO as those used in dissolving the SB203580 and Anisomycin, with 5 rats in each group. The changes of pain sensitivity were detected 3 days later. According to the 
experimental groups, 5 rats in each group were killed on the 1st or 10th day after CFA injection, and then the following experiments were conducted.

\section{Measurement of ankle circumference}

The circumference of affected ankle in rats was measured to judge the degree of local swelling of ankle arthritis. In quiet state, the rats were fixed to the self-made wooden rack. The ankle was gently surrounded by ordinary inelastic cotton thread to measure its circumference.

\section{Thermal hyperalgesia}

The hot plate was maintained at $55^{\circ} \mathrm{C} \pm 0.5^{\circ} \mathrm{C}$ and the rats were placed on it to carry on the thermal hyperalgesia. The pain threshold was determined by the paw withdrawal latency (PWL). The maximum cutoff time of each animal was $15 \mathrm{~s}$. Three experiments were carried out at an interval of 5-10 min per claw. The average latency of paw withdrawal of each claw was calculated, and the average value of affected claw was subtracted from the other claw, and the obtained value represented hyperalgesia of affected claw.

\section{Paw withdrawal threshold (PWT)}

The threshold of mechanical paw withdrawal of rat soles was determined by von Frey filaments. Different weights of filaments were used to act on the central part of the soles of rats to induce claw contraction. The filaments causing claw contraction was used to represent the PWT. The maximum cutoff time of each animal was $15 \mathrm{~s}$.

\section{Hematoxylin and eosin (HE) staining}

After modeling and deep anesthesia [intraperitoneal injection of $10 \%$ urethane $(1 \mathrm{~g} / \mathrm{kg})$ ], the rats were killed by cervical dislocation and the two-sided ankle was mutilated for conducting histopathological examination. After removing the skin of ankle, the ankle was decalcified in the decalcanizer for $12 \mathrm{~h}$, and then the tissue block was embedded with paraffin and then sectioned. After that, the sections were used to perform HE staining, and the changes of synovial structure, cartilage and degree of bone damage after inflammation were observed under microscope.

\section{Safranin-0/Fast Green staining}

After mutilation, the ankle was decalcified in the $20 \%(\mathrm{v} / \mathrm{v})$ ethylene diamine tetraacetic acid (EDTA) solution ( $\mathrm{pH} \mathrm{7.2)} \mathrm{and} \mathrm{then} \mathrm{dissected} \mathrm{in} \mathrm{sagittal} \mathrm{plane.} \mathrm{The} \mathrm{ankle} \mathrm{was} \mathrm{processed} \mathrm{in} \mathrm{the} \mathrm{Tissue-Tek} \mathrm{VIP}$ 1000 tissue processor (Miles, Inc., Elkhart, IN) and embedded in a single Paraplast X-tra (Thermo Fisher Scientific Inc. (Waltham, Mass). The slices were cut into $6-\mu \mathrm{m}$ sections and installed on the slide for performing Safranin-0/Fast Green staining to evaluate the severity of cartilage injury.

\section{Immunohistochemistry}

The tissue sections were dewaxed to hydration, reacted in $92-98^{\circ} \mathrm{C} 10 \mathrm{~g} / \mathrm{L}$ citric acid-sodium citrate solution $(500 \mathrm{~mL})$ for $15 \mathrm{~min}$, incubated with hydrogen peroxide $(0.882 \mathrm{~mol} / \mathrm{L})$ at room temperature for 
10 min, and washed with PBS for 3 times. Then, the sections were sealed by sealing fluid containing 100 $\mathrm{mL} / \mathrm{L}$ bovine serum, incubated at room temperature for $1 \mathrm{~h}$, added with primary antibodies TNF- $\mathrm{a}(1: 100$, ab6671, Abcam, Cambridge, MA, USA) and IL-1 $\beta$ (1:100, ab9722, Abcam) for incubation at $4^{\circ} \mathrm{C}$ overnight, followed by PBS washing for 3 times and incubation with secondary antibody IgG (1:2000, ab205718, Abcam) at $37^{\circ} \mathrm{C}$ for $30 \mathrm{~min}$. Subsequently, the sections were developed by diaminobenzidine (DAB), washed by distilled water, re-stained, dehydrated, cleaned, sealed by neutral gum, and observed under microscope. The image was analyzed by Image-Pro Plus 6.0 software.

\section{Enzyme-linked immunosorbent assay (ELISA)}

The rats were anesthetized by inhalation of ether, and then the blood sample was taken from orbit (blood sample was collected on the 1st and 10th day, respectively). After proper treatment, the concentrations of TNF-a (RTA00) and IL-1 $\beta$ (RLB00) in serum were measured by ELISA kit (R\&D Systems, Abingdon, United Kingdom). The rat serum was added into the ELISA plates and incubated with the coating buffer at $37^{\circ} \mathrm{C}$ for $2 \mathrm{~h}$ and then with the $10 \%$ calf serum at $4^{\circ} \mathrm{C}$ overnight. Then, the blood samples were cultured with the primary antibody at $37^{\circ} \mathrm{C}$ for $2 \mathrm{~h}$ and the secondary antibody for $1 \mathrm{~h}$, The termination solution was added after the chromatogenic reaction. The optical density of each well was measured at $450 \mathrm{~nm}$.

\section{Western blot analysis}

The protein of ankle joint tissues of rats was extracted and the concentration of protein was determined according to the instructions of BCA kit (Thermo Scientific Pierce; Rockford, IL). The extracted protein was added with sample loading buffer and boiled at $95^{\circ} \mathrm{C}$ for $10 \mathrm{~min}$. Protein sample was separated via sodium dodecyl sulfate-polyacrylamide gel electrophoresis (SDS-PAGE) $(10 \%[\mathrm{w} / \mathrm{v}])$ with the voltage change from $80 \mathrm{v}$ to $120 \mathrm{v}$ (wet transfer, voltage of $100 \mathrm{mv}, 45-70 \mathrm{~min}$ ). The polyvinylidene fluoride (PVDF) membrane was sealed by $5 \%$ BSA at room temperature for $1 \mathrm{~h}$ and incubated with the primary antibodies p38 (1:1000, ab31828, Abcam), Pp38 (1:1000, ab47363, Abcam), $\beta$-actin (1:5000, ab32572, Abcam) and ASK1 (1:1000, ab45178, Abcam) at $4^{\circ} \mathrm{C}$ overnight. After TBST washing for 3 times, the membrane was incubated with the secondary antibody IgG (1:2000, ab205718, Abcam) at room temperature for $1 \mathrm{~h}$, followed by TBST washing. After that, the membrane was developed by enhanced chemiluminescence (ECL) method and imaged by Bio-OAdGelEZ imager (Bio-OAd, California, USA). The target band was analyzed by Image $\mathrm{J}$ software (National Institutes of Health, Bethesda, Maryland, USA). The relative expression of protein was shown as the gray value of target protein/the gray value of $\beta$-actin, with $\beta$-actin as the internal reference.

\section{Statistical analysis}

All data analyses were conducted using SPSS 21.0 software (IBM Corp. Armonk, NY, USA). All the data were consistent with normal distribution by Kolmogorov-SmiRnov test, and expressed as mean \pm standard deviation. Comparison of data between two groups was conducted by $t$-test, while comparison among multiple groups was conducted by one-way analysis of variance (ANOVA). After the ANOVA analysis, comparisons between two data were conducted by Tukey's-multi-comparisons test. $P$ is a twosided test, values of $P<0.05$ were considered statistically significant. 


\section{Results}

\section{Successful induction of MA models}

After $8 \mathrm{~h}$ of CFA induction, rats with arthritis showed obvious changes in physical signs, the affected side showed obvious swelling. One day after inflammation, the degree of local redness and swelling reached the peak. Uninjured side showed no significant change (Figure 1A). The circumference of affected ankle at day 1-10 was significantly longer than the sham group (Figure 1B). Pathological examination of bilateral ankle in rats at day 1 and day 10 after inflammation was conducted, and the results presented that one day after inflammation (24 h), there were obvious pathological changes in the joint cavity, the joint cavity space became narrow and synovial bursa became rough. A large number of inflammatory cell infiltration was observed under microscope. On the 10th day after inflammation, the local inflammatory infiltration was significantly improved and the inflammatory cells were significantly decreased, but still significantly higher than those on the uninjured side (Figure $1 C$ and $D$ ). The results of animal behavior test showed that there was no significant difference in basic PWT and PWL before inflammation in different treatment groups. Four hours after inflammation, thermal hyperalgesia and tactile pain began to occur in the posterior claw of the affected side (Figure 1E).

\section{DEX could reduce thermal hyperalgesia}

Intraabdominal injection of low dose $(2.5 \mu \mathrm{g} / \mathrm{kg})$ of DEX did not prolong the PWL and PWT of the affected side, while $5,10,20 \mu \mathrm{g} / \mathrm{kg}$ of DEX obviously prolong PWL and PWT of affected side of MA rats. In addition, the analgesic effect of DEX increased in a dose-dependent manner, and the analgesic time was prolonged in a dose-dependent manner. The analgesic effect of DEX reached the peak at $60 \mathrm{~min}$ after administration (Figure 2A). After 10 days of DEX administration, HE staining showed that inflammatory cell infiltration decreased with the increase of DEX concentration (Figure 2B). The circumference of affected ankle was improved with the increase of concentration of DEX (Figure 2C). Thus, we selected $20 \mu \mathrm{g} / \mathrm{kg}$ of DEX to carry on the experiment.

Induction of MA could lead to activation of P38MARK signaling pathway and aggravated the expression of inflammatory factors

ELISA was used to detect the serum levels of TNF- $\alpha$ and IL-1 $\beta$ (Figure 3A), the results revealed that after 1 day of CFA induction, the serum levels of TNF- $\alpha$ and IL-1 $\beta$ of rats were significantly increased, and improved after 10 days, but still higher than those in the sham group. Immunohistochemistry showed that on the 10th day after CFA induction, the expression of inflammatory factors decreased, but it was still higher than that in the sham group (Figure 3B).

p38MARK signaling pathway was considered to be important for the induction and maintenance of chronic inflammation [14]. Western blot analysis was applied to detect the protein expression of p38MARK signaling pathway-related factors (p38, p-p38 and ASK1), the results showed that the protein expression of ASK1, p-p38, and p38 increased significantly on the 1st day after CFA induction, and ASK1, 
p-p38, and p38was still highly expressed on the 10th day (Figure 3C). These results suggested that induction of MA could lead to activation of p38MARK signaling pathway and aggravated the expression of inflammatory factors.

\section{Inhibition of p38MARK signaling pathway could reduce hyperalgesia and inflammation in MA rats}

SB203580 is a p38MARK signaling pathway inhibitor that effectively inhibits kinase activity in the signaling pathway. We treated MA rats with SB203580 through intraabdominal injection. It was found that the expression of ASK1, p-p38, and p38 in rats was significantly decreased (Figure 4A), and the PWT, and PWL in affected side was significantly prolonged (Figure 4B). HE staining showed decrease of inflammatory cell infiltration in ankle of rats and serum levels of inflammatory factors (Figure $4 C$ and D). The above findings revealed that inhibition of p38MARK signaling pathway could reduce hyperalgesia and inflammation in MA rats.

\section{DEX could inhibit p38MARK signaling pathway and alleviate inflammatory factor expression and hyperalgesia in MA rats}

After determining the therapeutic effect of DEX on hyperalgesia in MA rats and the activation of p38MARK signaling pathway after CFA induction in rats, we speculated that the treatment of DEX would affect the p38MARK signaling pathway. So, we detected p38MARK signaling pathway-related proteins in rats and found that DEX could inhibit the expression of ASK1, p-p38, and p38. It is reported that Anisomycin can effectively activate intracellular p38mark signaling pathway [15]. The expression of ASK1, p-p38, and p38 in MA rats increased significantly after treatment with Anisomycin, and the increase of ASK1, p-p38, and p38 was inhibited when treated with DEX (Figure 5A). The results of inflammatory factor detection and HE staining were the same (Figure 5B and C). The results of PWT and PWL showed that the PWT and PWL were significantly shortened after the treatment of Anisomycin, while the PWT and PWL were significantly prolonged after DEX treatment (Figure 5D). The results showed that DEX could inhibit p38MARK signaling pathway and alleviate inflammatory factor expression and hyperalgesia in MA rats.

\section{Discussion}

Inflammatory joint diseases are painful diseases that are particularly popular in the old people; with life expectancies increasing, more and more people will be affected by arthritis [16]. Current treatments designed to relieve symptoms are effective in some patients, but they can have adverse side effects or high costs [17]. Therefore, it is very urgent to develop better therapeutics to manage and ultimately cure arthritis. In the current study, a CFA-induced MA rat model was established to investigate the effect of DEX on hyperalgesia with the involvement of the p38MAPK signaling pathway. It was proved that administration of $20 \mu \mathrm{g} / \mathrm{kg}$ DEX could alleviate hyperalgesia, decrease the expression of TNF- $\alpha$ and IL-1 $\beta$ and inhibit the activation of the p38MAPK signaling pathway in CFA-induced MA rats. 
The CFA-induced MA rat model, which shows many of the pathological features of RA, has been widely deemed as a laboratory model in the study of arthritic pain [9]. After CFA induction, affected side showed obvious swelling, thermal hyperalgesia and tactile pain, increased serum levels of TNF- $\alpha$ and IL-1 $\beta$, and activated the p38MAPK signaling pathway (increased expression of ASK1, p-p38 and p38). As presented by previous studies, intraarticular injection of CFA could cause knee swelling and increased levels of TNFa $[18,19]$. In line with our study, Xu B et al also pointed out that unilateral intraarticular injection of CFA could result in obvious joint inflammation and thermal hyperalgesia [20]. The inflammation induced by the injection of CFA could cause the induction of p-p38 MAPK in the dorsal horn [21].

In addition, the role of the p38MAPK signaling pathway in hyperalgesia and inflammation in CFA-induced MA rat models was also investigated. The results clarified that inhibition of p38MARK signaling pathway could reduce hyperalgesia and inflammation in CFA-induced MA rats. It is reported that p38MAPK signal transduction pathway is activated by cellular stress and pro-inflammatory cytokines and plays a key role in inflammatory responses [22]. Inflammatory mediators such as pro-inflammatory cytokines and chemokines are produced during inflammation, which can evoke pain by directly activating and sensitizing nociceptors [23]. In the spinal cord, the levels of some pro-inflammatory factors such as cyclooxygenase-2, IL-1 $\beta$, vanilloid receptor-1 are upregulated by p38MAPK [21]. Pervious evidence presented that suppression of p38MAPK phosphorylation in the dorsal root ganglion could prevent the development of pain hypersensitivity in various pathological pain scenarios [24]. In line with our study, pervious study showed that administration of inhibitor of p38MAPK could effectively alleviate inflammation and arthritis [25].

To further explore the related mechanism, we found that DEX could inhibit p38MARK signaling pathway and alleviate inflammatory factor expression and hyperalgesia in CFA-induced MA rats. DEX is proved to exert dose-dependent analgesic effects against inflammation as well as neuropathic and post-operative pain [3]. DEX was demonstrated to suppress thermal hyperalgesia and spinal glial activation in rat model of MA [6]. p38 MAPK is mainly activated within cells, which in turn triggers the produce of key inflammatory factors, such as TNFa, and IL-1; this production can play a crucial role in the pathogeny of some chronic inflammatory diseases, such as RA $[26,27]$. DEX could peripherally prevent the phosphorylation of p38 MAPK when used as an adjunct to local anesthetics via inhibition of inflammation and TNF-a production [28]. The MAPK signaling pathway, including ERK, JNK, and P38 signals, were also analyzed in one previous study, and the results suggested that the activation of these three signals were obviously suppressed by the administration of DEX [29]. Partly consistent with our result, Ji D et al demonstrated that DEX could exert anti-nociceptive effect on a rat model of MA via suppression of the TLR4/NF-KB p65 signaling pathway [3].

To sum up, we were the first to reveal that DEX suppressed the activation of the p38MARK signaling pathway, and consequently reduced hyperalgesia and inflammation in CFA-induce MA rats. Our results provided essential evidence to further understand the protective mechanism of DEX in MA, and hint the possibility of p38MARK pathway as a novel target for MA patients. Based on our findings, more researches are warranted to determine reasonable regimens for MA patients and to clarify the adverse 
effects of DEX in the management of MA. In the future, we shall carry out more prospective trials to transition our findings to clinical applications.

\section{Declarations}

\section{Acknowledgements}

Not applicable.

\section{Funding}

Not applicable.

\section{Availability of data and materials}

All the data generated or analyzed during this study are included in this published article.

\section{Competing interests}

All authors declare that there is no conflict of interests in this study.

\section{Author Contributions}

$\mathrm{BN}$ is the the guarantor of integrity of the entire study; $\mathrm{BN}$ contributed to the study design and manuscript preparation; BN and QL contributed to the study concepts and experimental studies; $\mathrm{BN}$ and $\mathrm{HJ}$ contributed to the definition of intellectual content; $\mathrm{BN}, \mathrm{HJ}$ and $\mathrm{HC}$ contributed to the literature research; $\mathrm{HJ}$ and $\mathrm{HC}$ contributed to the clinical studies; $\mathrm{HC}$ and QL contributed to the data acquisition; $\mathrm{BN}, \mathrm{HC}$ and QL contributed to the data analysis; QL statistical analysis; All authors read and approved the final manuscript.

\section{References}

1. Hong Y, Palaksha K, Reproductive SLJ, et al. Morphological Changes of Bones and Joints with Rheumatoid Arthritis and Osteoarthritis. 2011;35: 143-149.

2. Mapp PI,Walsh DA. Mechanisms and targets of angiogenesis and nerve growth in osteoarthritis. Nat Rev Rheumatol. 2012;8: 390-398. https://doi.org/10.1038/nrrheum.2012.80.

3. Ji D, Zhou Y, Li S, et al. Anti-nociceptive effect of dexmedetomidine in a rat model of monoarthritis via suppression of the TLR4/NF-kappaB p65 pathway. Exp Ther Med. 2017;14: 4910-4918. https://doi.org/10.3892/etm.2017.5196.

4. Chien SY, Huang $\mathrm{CY}$, Tsai $\mathrm{CH}$, et al. Interleukin-1 beta induces fibroblast growth factor 2 expression and subsequently promotes endothelial progenitor cell angiogenesis in chondrocytes. Clin Sci (Lond). 2016;130: 667-681. https://doi.org/10.1042/CS20150622. 
5. Shimode N, Fukuoka T, Tanimoto $\mathrm{M}$, et al. The effects of dexmedetomidine and halothane on Fos expression in the spinal dorsal horn using a rat postoperative pain model. Neurosci Lett. 2003;343: 45-48. https://doi.org/10.1016/s0304-3940(03)00309-4.

6. Xu B, Zhang WS, Yang JL, et al. Dexmedetomidine blocks thermal hyperalgesia and spinal glial activation in rat model of monoarthritis. Acta Pharmacol Sin. 2010;31: 523-530. https://doi.org/10.1038/aps.2010.32.

7. Motawea HKB, Chotani MA, Ali M, et al. Human Placenta Expresses alpha2-Adrenergic Receptors and May Be Implicated in Pathogenesis of Preeclampsia and Fetal Growth Restriction. Am J Pathol. 2018;188: 2774-2785. https://doi.org/10.1016/j.ajpath.2018.08.011.

8. Patel R, Qu C, Xie JY, et al. Selective deficiencies in descending inhibitory modulation in neuropathic rats: implications for enhancing noradrenergic tone. Pain. 2018;159: 1887-1899. https://doi.org/10.1097/j.pain.0000000000001300.

9. Zhang WS, Xu H, Xu B, et al. Antihyperalgesic effect of systemic dexmedetomidine and gabapentin in a rat model of monoarthritis. Brain Res. 2009;1264: 57-66.

https://doi.org/10.1016/j.brainres.2009.01.029.

10. Hajialilo M, Ghorbanihaghjo A, Valaee L, et al. A double-blind randomized comparative study of triamcinolone hexacetonide and dexamethasone intra-articular injection for the treatment of knee joint arthritis in rheumatoid arthritis. Clin Rheumatol. 2016;35: 2887-2891. https://doi.org/10.1007/s10067-016-3397-4.

11. Wang ZC, Lu H, Zhou Q, et al. MiR-451 inhibits synovial fibroblasts proliferation and inflammatory cytokines secretion in rheumatoid arthritis through mediating p38MAPK signaling pathway. Int $\mathrm{J}$ Clin Exp Pathol. 2015;8: 14562-14567.

12. Liu S, Feng G, Wang GL, et al. p38MAPK inhibition attenuates LPS-induced acute lung injury involvement of NF-kappaB pathway. Eur J Pharmacol. 2008;584: 159-165. https://doi.org/10.1016/j.ejphar.2008.02.009.

13. Clark AR,Dean JL. The p38 MAPK Pathway in Rheumatoid Arthritis: A Sideways Look. Open Rheumatol J. 2012;6: 209-219. https://doi.org/10.2174/1874312901206010209.

14. Schett G, Zwerina J,Firestein G. The p38 mitogen-activated protein kinase (MAPK) pathway in rheumatoid arthritis. Ann Rheum Dis. 2008;67: 909-916. https://doi.org/10.1136/ard.2007.074278.

15. Wood MW, Segal JA, Mark RJ, et al. Inflammatory cytokines enhance muscarinic-mediated arachidonic acid release through p38 mitogen-activated protein kinase in A2058 cells. J Neurochem. 2000;74: 2033-2040. https://doi.org/10.1046/j.1471-4159.2000.0742033.x.

16. Helmick CG, Felson DT, Lawrence RC, et al. Estimates of the prevalence of arthritis and other rheumatic conditions in the United States. Part I. Arthritis Rheum. 2008;58: 15-25. https://doi.org/10.1002/art.23177.

17. Shi M, Wang A, Prescott $D$, et al. Infection with an intestinal helminth parasite reduces Freund's complete adjuvant-induced monoarthritis in mice. Arthritis Rheum. 2011;63: 434-444. https://doi.org/10.1002/art.30098. 
18. Keeble J, Russell F, Curtis B, et al. Involvement of transient receptor potential vanilloid 1 in the vascular and hyperalgesic components of joint inflammation. Arthritis Rheum. 2005;52: 3248-3256. https://doi.org/10.1002/art.21297.

19. Kelso EB, Dunning L, Lockhart JC, et al. Strain dependence in murine models of monoarthritis. Inflamm Res. 2007;56: 511-514. https://doi.org/10.1007/s00011-007-7058-6.

20. Xu B, Zhang WS, Yang JL, et al. Evidence for suppression of spinal glial activation by dexmedetomidine in a rat model of monoarthritis. Clin Exp Pharmacol Physiol. 2010;37: e158-166. https://doi.org/10.1111/j.1440-1681.2010.05426.x.

21. Fang JQ, Du JY, Liang Y, et al. Intervention of electroacupuncture on spinal p38 MAPK/ATF-2/VR-1 pathway in treating inflammatory pain induced by CFA in rats. Mol Pain. 2013;9: 13. https://doi.org/10.1186/1744-8069-9-13.

22. Kumar S, Boehm J,Lee JC. p38 MAP kinases: key signalling molecules as therapeutic targets for inflammatory diseases. Nat Rev Drug Discov. 2003;2: 717-726. https://doi.org/10.1038/nrd1177.

23. Matsuda M, Huh Y,Ji RR. Roles of inflammation, neurogenic inflammation, and neuroinflammation in pain. J Anesth. 2019;33: 131-139. https://doi.org/10.1007/s00540-018-2579-4.

24. Ji RR, Gereau RWt, Malcangio M, et al. MAP kinase and pain. Brain Res Rev. 2009;60: 135-148. https://doi.org/10.1016/j.brainresrev.2008.12.011.

25. Boyle DL, Jones TL, Hammaker $D$, et al. Regulation of peripheral inflammation by spinal p38 MAP kinase in rats. PLoS Med. 2006;3: e338. https://doi.org/10.1371/journal.pmed.0030338.

26. Akhtari Z, Zaringhalam J, Eidi A, et al. Bidirectional effects of serum TNF alpha level and spinal p38MAPK phosphorylation on hyperalgesia variation during CFA-induced arthritis. EXCLI J. 2012;11: 373-385.

27. Cuenda A,Rousseau S. p38 MAP-kinases pathway regulation, function and role in human diseases. Biochim Biophys Acta. 2007;1773: 1358-1375. https://doi.org/10.1016/j.bbamcr.2007.03.010.

28. Yamakita S, Matsuda M, Yamaguchi Y, et al. Dexmedetomidine prolongs levobupivacaine analgesia via inhibition of inflammation and p38 MAPK phosphorylation in rat dorsal root ganglion. Neuroscience. 2017;361: 58-68. https://doi.org/10.1016/j.neuroscience.2017.08.011.

29. Wang Q, Tan Y, Zhang N, et al. Dexmedetomidine inhibits activation of the MAPK pathway and protects PC12 and NG108-15 cells from lidocaine-induced cytotoxicity at its maximum safe dose. Biomed Pharmacother. 2017;91: 162-166. https://doi.org/10.1016/j.biopha.2017.04.084.

\section{Figures}




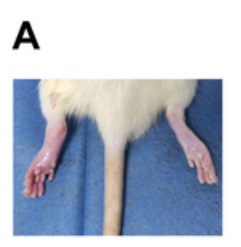

D

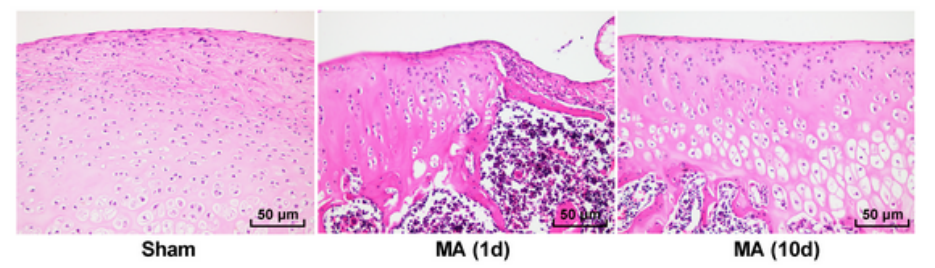

MA (1d)

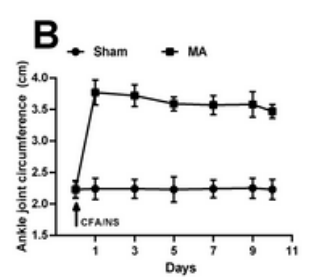

C

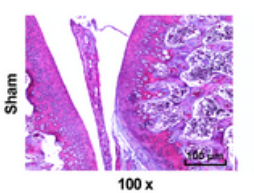

$100 \times$
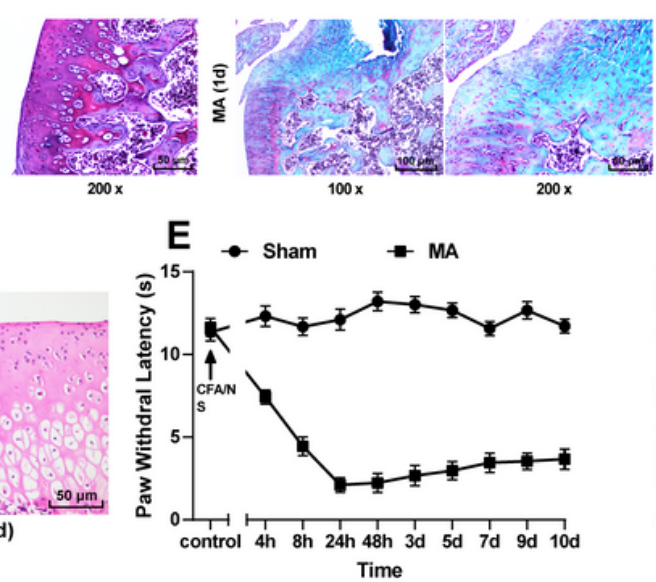

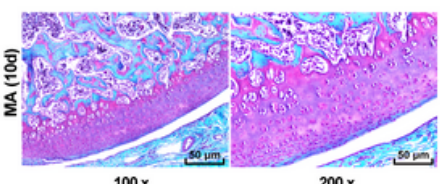

$100 x$

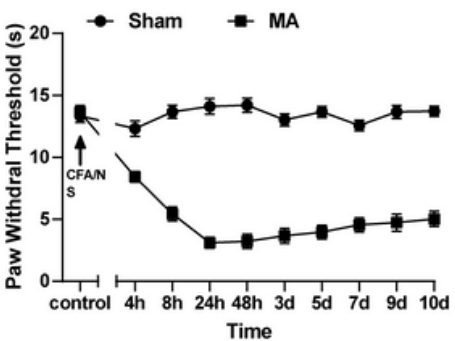

Figure 1

Establishment of CFA-induced ankle monoarthritis models. Notes: A, The injection of CFA in the unilateral ankle in rats resulted in swelling. The left foot was affected side and the right foot was not injected; $B$, Measurement of ankle circumference after CFA injection in rats, CFA/NS is the time point of CFA or saline injection; C, Safranin-O/Fast Green staining of ankle tissue sections in rats $(10 \times, 20 \times)$; D, HE staining of ankle tissue sections in rats; $\mathrm{E}$ : Test of PWL and PWT in rats after induction of CFA, CFA/NS is the time point of CFA or saline injection. 
A

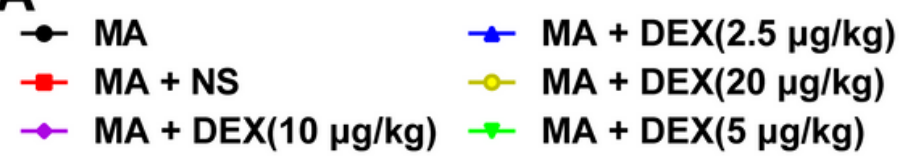

$$
\begin{array}{ll}
\rightarrow-M A & -M A+\operatorname{DEX}(2.5 \mu \mathrm{g} / \mathrm{kg}) \\
\rightarrow \mathrm{MA}+\mathrm{NS} & -\mathrm{MA}+\operatorname{DEX}(20 \mu \mathrm{g} / \mathrm{kg}) \\
\rightarrow \mathrm{MA}+\operatorname{DEX}(10 \mu \mathrm{g} / \mathrm{kg}) & \rightarrow \mathrm{MA}+\operatorname{DEX}(5 \mu \mathrm{g} / \mathrm{kg})
\end{array}
$$
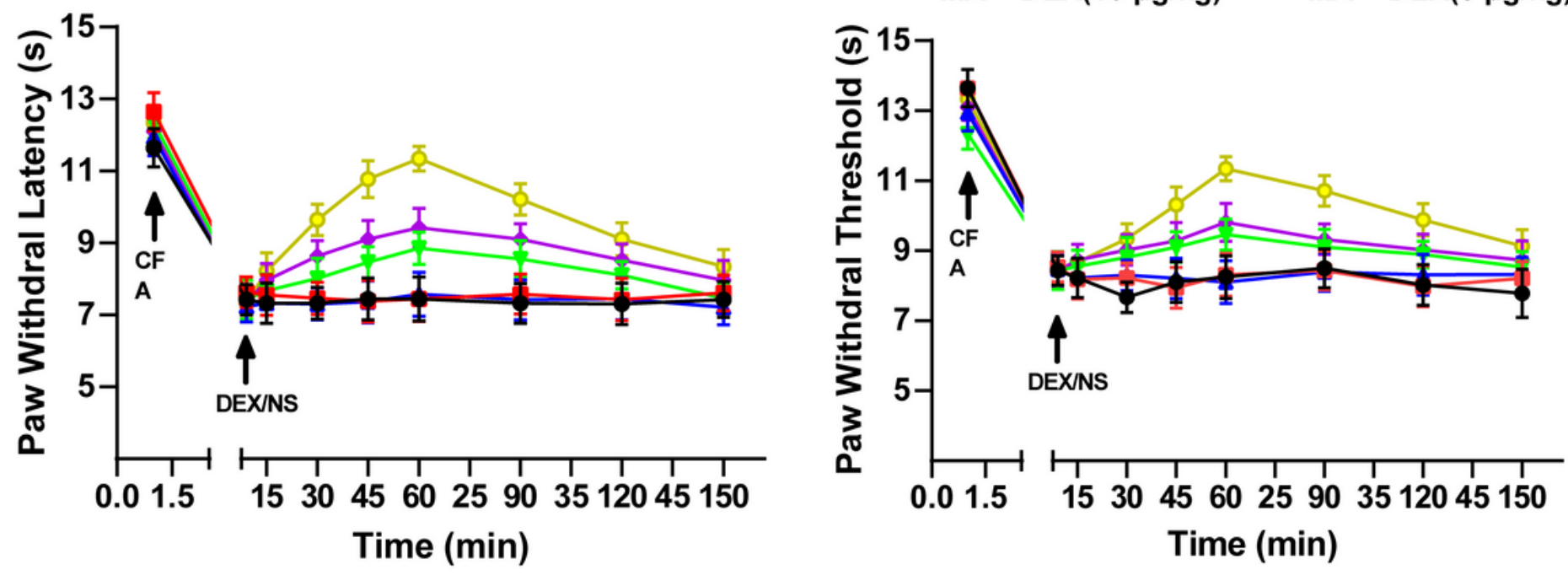

B
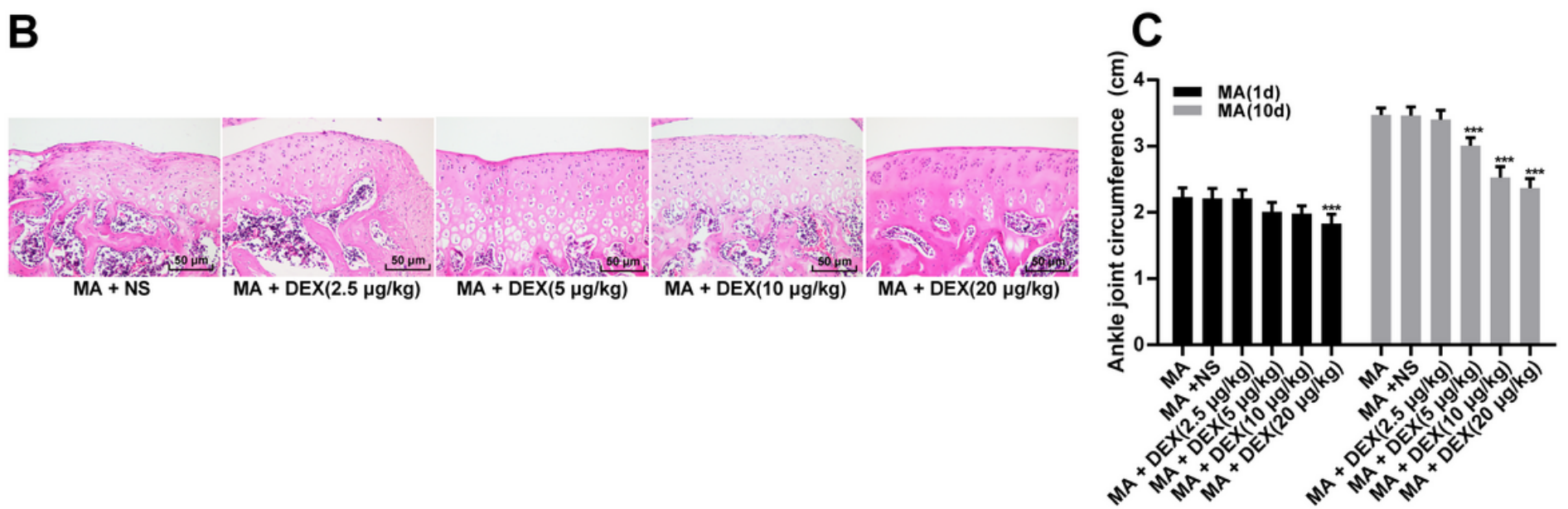

Figure 2

DEX could reduce thermal hyperalgesia. Notes: A, Measurement of PWL and PWT in MA rats after different concentrations of DEX injection; $B$, HE staining was applied to observe the pathological effects of different concentrations of DEX on ankle tissue in MA rats; $C$, Measurement of ankle circumference; compared with the MA group, $* \star \star ~ P<0.001$. 

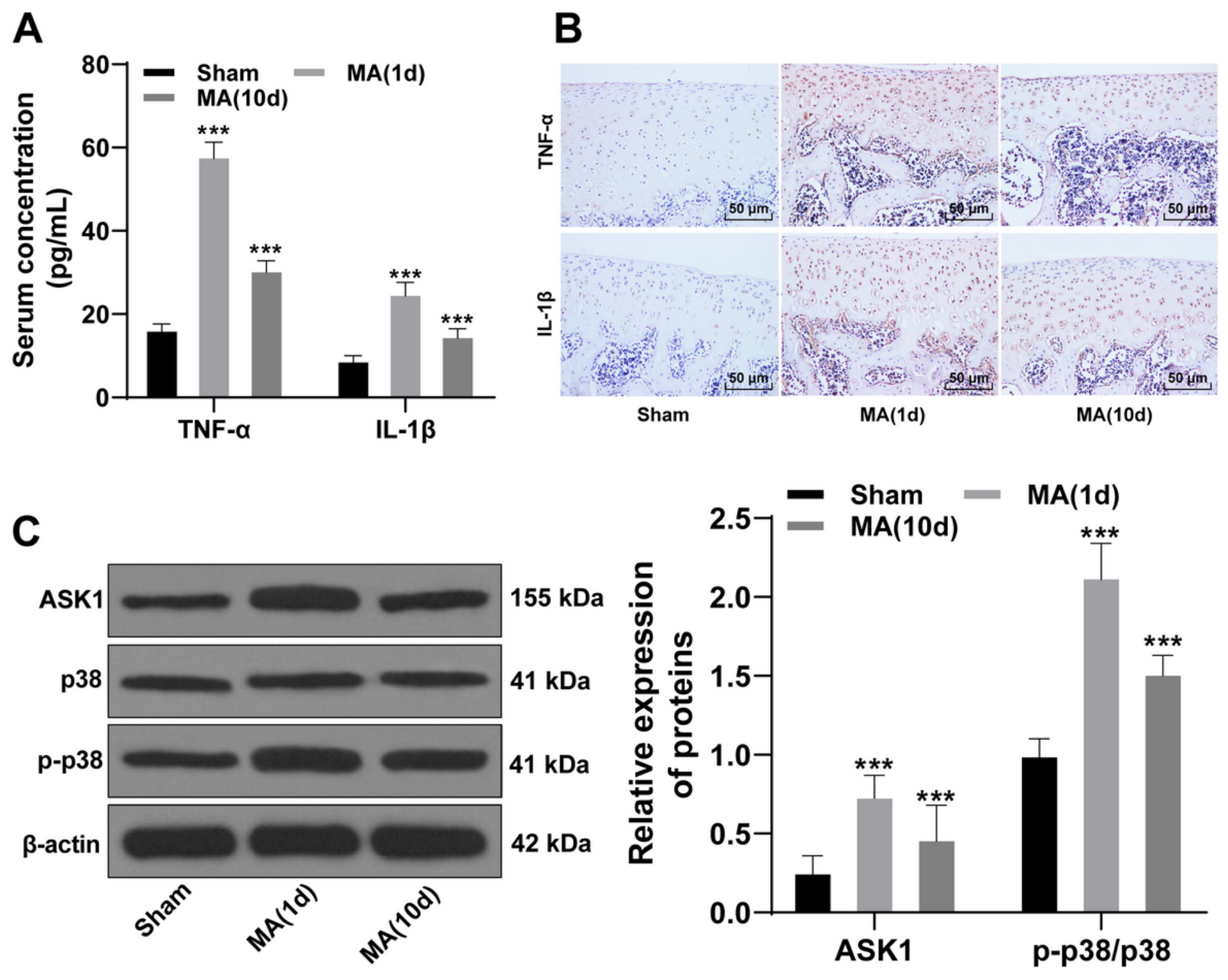

Figure 3

Induction of MA could lead to activation of p38MARK signaling pathway and aggravated the expression of inflammatory factors. Notes: A, Detection of the expression of TNF- $a$ and IL-1 $\beta$ in serum of rats on the 1 st and 10th day after CFA induction by ELISA, compared with the sham group, $\star \star \star ~ P<0.001 ; B$, Expression of TNF- $a$ and IL-1 $\beta$ in ankle of MA rats detected by IHC; $C$, Detection of the expression of ASK1, p-p38 and p38 of rats on the 1st and 10th day after CFA induction by western blot, compared with the sham group, ${ }^{\star \star \star} \mathrm{P}<0.001$. 

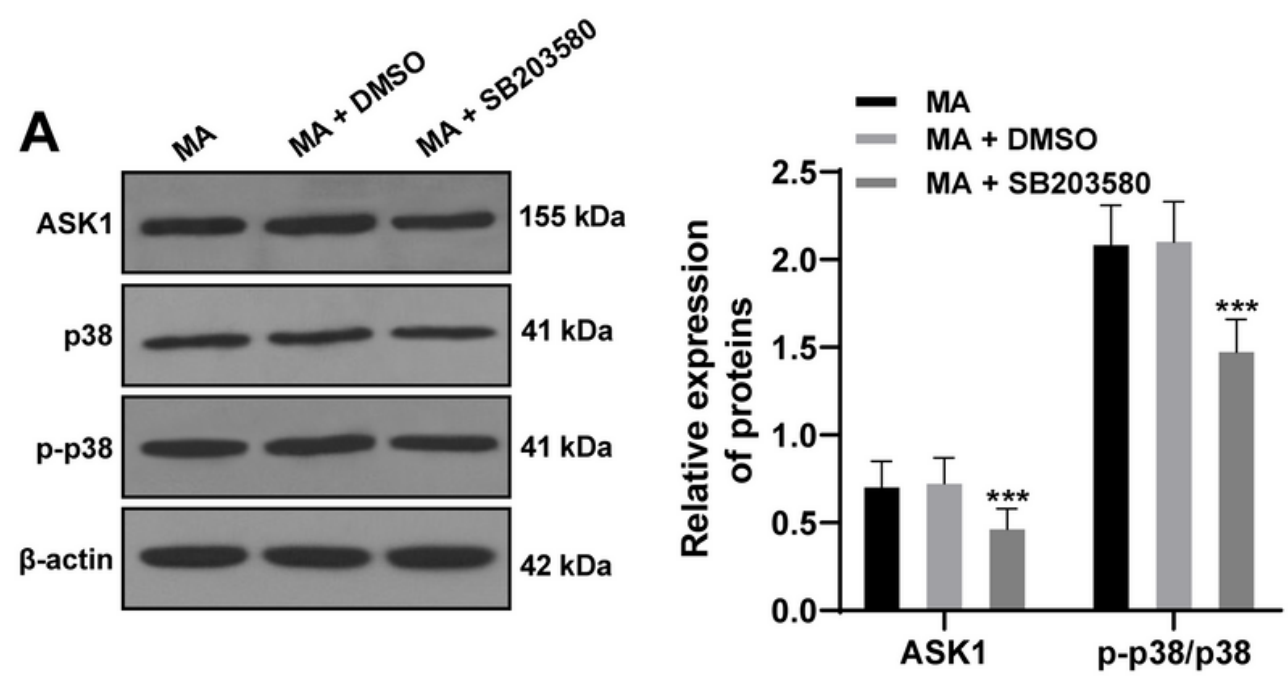

C

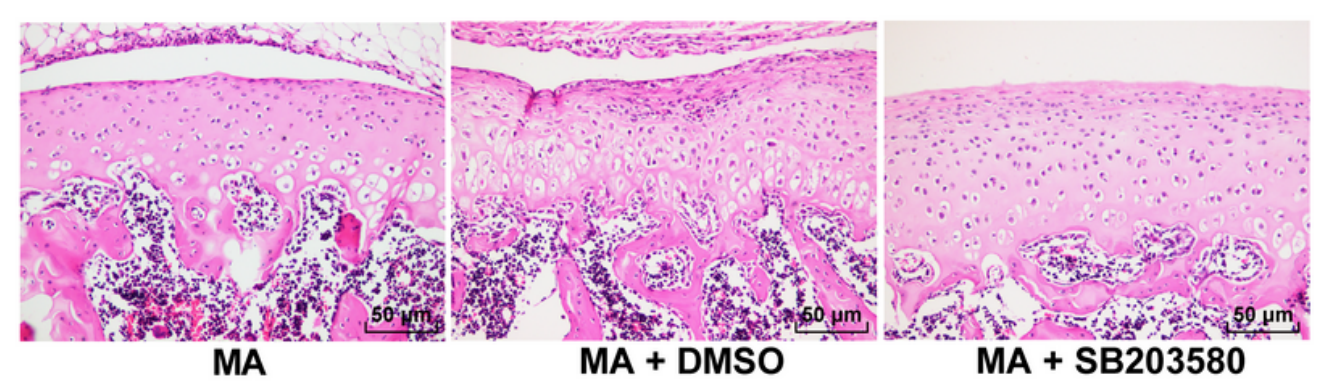

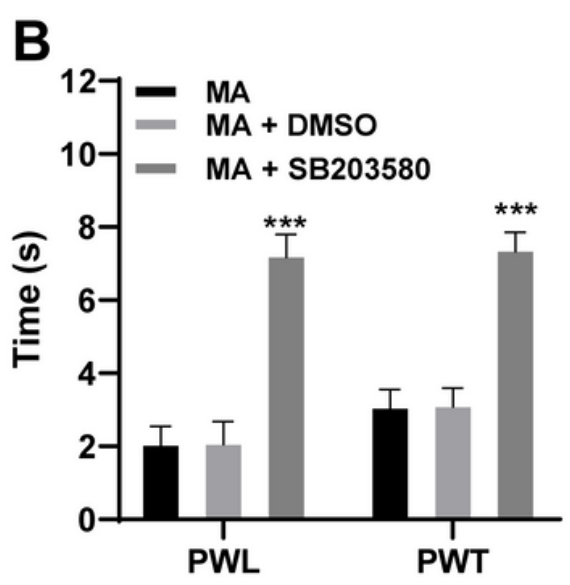

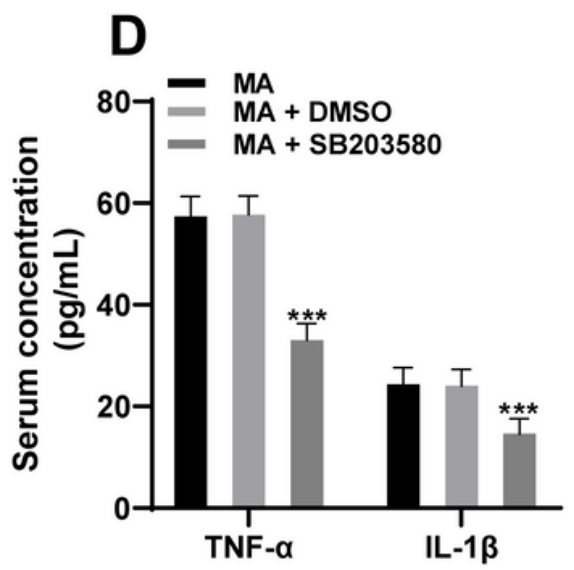

Figure 4

Inhibition of p38MARK signaling pathway could reduce hyperalgesia and inflammation in MA rats. Notes: A, Detection of the expression of ASK1, p-p38 and p38 of rats by western blot, compared with the MA group, $* \star * P<0.001 ; B, P W L$ and PWT in MA rats after SB203580 treatment, compared with the MA group, $\star \star \star P<0.001 ; C, H E$ staining was applied to observe the inflammatory cell infiltration after SB203580 treatment; D, Detection of the expression of TNF-a and IL-1 $\beta$ in serum of rats after SB203580 treatment, compared with the MA group, $* \star \star ~ P<0.001$. 

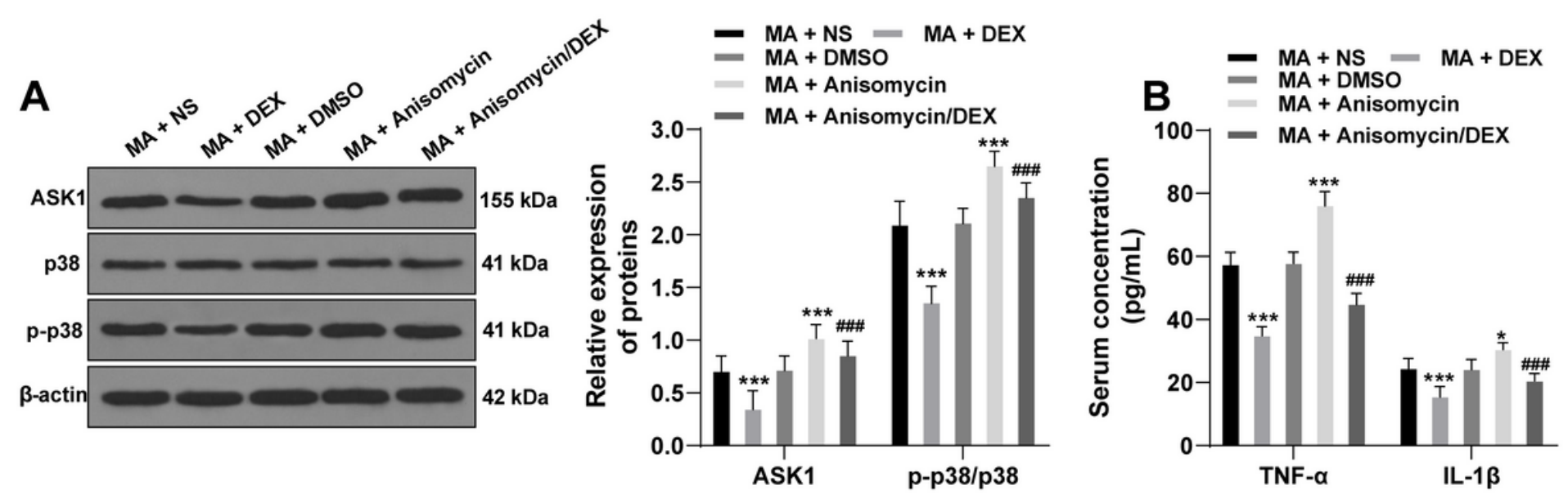

C
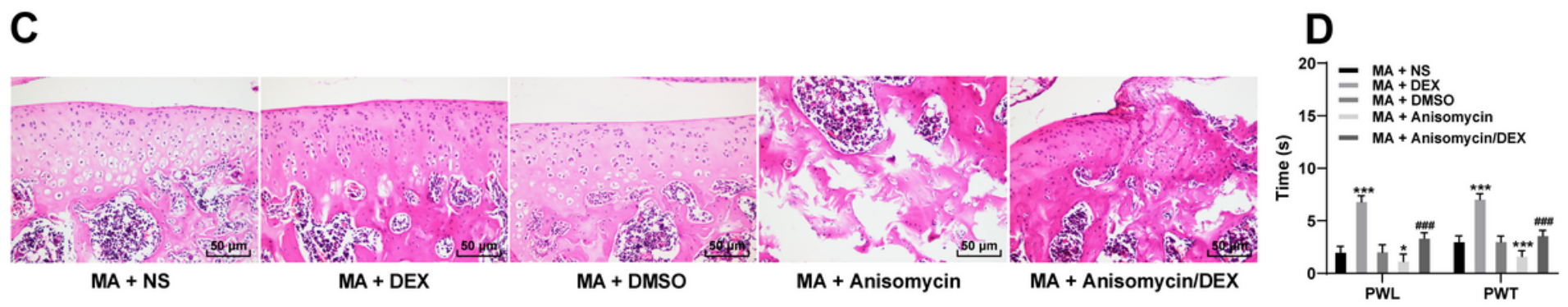

Figure 5

DEX could inhibit p38MARK signaling pathway and alleviate inflammatory factor expression and hyperalgesia in MA rats. Notes: A, Detection of the expression of ASK1, p-p38 and p38 of rats by western blot, compared with the MA group, ${ }^{\star \star *} \mathrm{P}<0.001$, compared with the MA + Anisomycin group, \#\#\# $\mathrm{P}<$ 0.001 ; B Detection of the expression of TNF- $a$ and IL-1 $\beta$ in serum of rats, compared with the MA group, *** $P<0.001$, compared with the MA + Anisomycin group, \#\#\# $\mathrm{P}<0.001 ; \mathrm{C}, \mathrm{HE}$ staining was applied to observe the inflammatory cell infiltration; $D, P W L$ and PWT in MA rats after different treatment, compared with the MA group, ${ }^{*} \mathrm{P}<0.05$, $* \star * \mathrm{P}<0.001$, compared with the $\mathrm{MA}+$ Anisomycin group, \#\#\# $\mathrm{P}<0.001$. 